\title{
Systemic Tremor Induced by Oxycodone Hydrochloride Controlled-Release Tablets in a Patient: A Case Report and Mechanism Exploration
}

\author{
Qirui Tai ${ }^{1{ }^{*},}$ Mili Shi ${ }^{1}$, Qiuyan Song ${ }^{1}$, Yijie Shi ${ }^{1}$, Zhiwei Tang ${ }^{2}$ \\ ${ }^{1}$ Sixth Affiliated Hospital of Kunming Medical University, Yuxi, Yunnan 653100, China. \\ ${ }^{2}$ First Affiliated Hospital of Kunming Medical University, Kunming, Yunnan 650000, China.
}

\begin{abstract}
How to cite this paper: Qirui Tai, Mili Shi, Qiuyan Song, Yijie Shi, Zhiwei Tang. (2021) Systemic Tremor Induced by Oxycodone Hydrochloride ControlledRelease Tablets in a Patient: A Case Report and Mechanism Exploration. International Journal of Clinical and Experimental Medicine Research, 5(3), 249-253.

DOI: 10.26855/ijcemr.2021.07.003
\end{abstract}

Received: March 30, 2021

Accepted: April 25, 2021

Published: May 11, 2021

*Corresponding author: Qirui Tai, Sixth Affiliated Hospital of Kunming Medical University, Yuxi, Yunnan 653100, China.

Email: taiqr@163.com

\begin{abstract}
Rationale: ORCT drug instructions display, OCRT can induce a rare adverse reaction of systemic tremor. Patient after taking a series of drugs and OCRT, the patient experienced adverse event of systemic tremor. Hence, we hypothesize that tremor is caused by OCRT. Patient concerns: An 83-year-old male patient a hard mass sized about $5 \mathrm{~cm} \times 3 \mathrm{~cm}$ could be touched on the inner side of anterior superior spine of left lower abdomen, local tenderness. Diagnosis: 1 . Abdominal distension and poor appetite to be checked; 2. Previous operation for bladder cancer; 3. Acute exacerbation of chronic obstructive pulmonary disease; 4. Arteriosclerotic encephalopathy; 5. Prostatic hyperplasia; and 6. Sleep disorders. Interventions: After hospitalization, the patient was given symptomatic treatment with Corydalis Yanhusuo analgesic pills and Shubitong capsules. On October 29, the patient's pain was still poorly controlled, so OCRT (10 mg, q12h PO) was given. On November 2, the dose of OCRT was adjusted to $20 \mathrm{mg}$, q12h PO. At 13:00 on November 3, the patient suffered from dizziness and systemic tremor for more than $5 \mathrm{~h}$. On November 4, the patient suffered from dizziness and systemic tremor, without apparent neurological signs. Outcomes: On November 4, OCRT was discontinued, and morphine sulfate controlled-release tablets (30 mg, q12h PO) were administered. MRI examination: No abnormal findings on craniocerebral DWI scans; EEG examination: Abnormal findings, epilepsy was excluded temporarily. On November 4, the tremor in patient was improved. On November 5, the patient no longer complained of tremor. Lessons: Correlation analysis between the tremor and the case suggested that the tremor was an adverse reaction caused by OCRT. According to our analysis, relevant mechanism of action was probably associated with the lowered secretion of $\gamma$-GABA and NE caused by the action of OCRT on $\mu$ receptors, which led to rhythmic, involuntary tremor of muscles in one or more functional areas. Or it may be that the OCRT caused excessive production of 5-HT to induce the 5-HT syndromic tremor.
\end{abstract}

\section{Keywords}

Oxycodone, Tremor, Mechanism, Locus Coeruleus, Mreceptors, Gene Polymorphism 


\section{Introduction}

Oxycodone (14-hydroxy-7, 8-dihydrocodeinone) is a novel semi-synthetic derivative of the baine, which is a full agonist of $\mu$ and $\kappa$ receptors [1]. With good sedative, analgesic, antitussive and antianxietic activities, it is clinically applied in the treatment of moderate to severe pain. Adverse reactions caused by oxycodone are primarily gastrointestinal impairments, with the most frequent being constipation, followed by nausea, vomiting, dizziness, headaches, drowsiness, anorexia, dysuria, itching of the skin, etc. There have been a few reports that the oxycodone can lead to respiratory depression, bone marrow suppression, liver injury, as well as anal and circulatory failure [2-4]. An adverse reaction of systemic tremor to oral OCRT is very rare, which has never been reported so far [5]. In this paper, a case of an elderly patient in our hospital developing systemic tremor after OCRT application is analyzed, in order to provide a reference for the adverse reaction monitoring of the drug and to promote rational medication in the clinical practice.

\section{Clinical data}

An 83-year-old male patient was admitted to our emergency department on October 21, 2019 due to "abdominal bloating and poor appetite for 1 week". Physical examination on admission: $\mathrm{T}=36.6^{\circ} \mathrm{C}, \mathrm{P}=95 \mathrm{bpm}, \mathrm{R}=20 / \mathrm{min}$, $\mathrm{BP}=117 / 72 \mathrm{mmHg}$. A hard mass sized about $5 \mathrm{~cm} \times 3 \mathrm{~cm}$ could be touched on the inner side of anterior superior spine of left lower abdomen, which was characterized by poor activity, clear medial boundaries and local tenderness. Diagnosis on admission: 1 . Abdominal distension and poor appetite to be checked; 2 . Previous operation for bladder cancer; 3. Acute exacerbation of chronic obstructive pulmonary disease; 4. Arteriosclerotic encephalopathy; 5. Prostatic hyperplasia; and 6. Sleep disorders. The patient had taken prolonged oral clonazepam for previous sleep disorders. He denied any history of drugs or food allergies. After hospitalization, the patient was given symptomatic treatment with compound amino acid (18AA-VII), enteral nutrition suspension, compound digestive enzyme capsules, Corydalis Yanhusuo analgesic pills and Shubitong capsules. On October 22, wheezing was heard in the lungs of patient, who was given additional compound bamboo saps and doxofylline injection. On October 23, the patient complained difficulty of excrement, so lactulose oral solution was given additionally. On October 24, the patient still had pain in the left lower abdomen, Yanhusuo analgesic pills were discontinued, and celecoxib capsules (200 mg, bid PO) were administered. On October 25, rabeprazole sodium enteric-coated capsules and budesonide/formoterol inhaler were administered additionally. On October 29, the patient's pain was still poorly controlled, so OCRT (10 mg, q12h PO) was given, as well as methylcobalamin tablets. On October 30, the patient felt nausea and vomiting, who was thus given metoclopramide tablets and injection. On November 2, the dose of OCRT was adjusted to $20 \mathrm{mg}$, q12h PO. At 13:00 on November 3, the patient suffered from dizziness and systemic tremor for more than $5 \mathrm{~h}$. $\mathrm{T}=37.0^{\circ} \mathrm{C}, \mathrm{P}=107 \mathrm{bpm}, \mathrm{R}=24 / \mathrm{min}, \mathrm{BP}=153 / 81 \mathrm{mmHg}, \mathrm{K}^{+}=4.23 \mathrm{mmol} / \mathrm{L}, \mathrm{Na}^{+}=135 \mathrm{mmol} / \mathrm{L}$, $\mathrm{Cl}^{-}=93 \mathrm{mmol} / \mathrm{L}$. On November 4, the patient suffered from dizziness and systemic tremor, without apparent neurological signs. Accordingly, OCRT was discontinued, and morphine sulfate controlled-release tablets (30 mg, q12h PO) were administered, as well as enteral nutrition suspension, metoclopramide tablets, rabeprazole sodium enteric-coated capsules, compound bamboo saps, compound digestive enzyme capsules and Shubitong capsules. The possibility of epileptic seizure was not considered after consultation in the Neurology Department. MRI examination: No abnormal findings on craniocerebral DWI scans; EEG examination: Abnormal findings, epilepsy was excluded temporarily. On November 4, the tremor in patient was improved. On November 5, the patient no longer complained of tremor. The study protocol was approved by the Ethics Committee of Sixth Affiliated Hospital of Kunming Medical University on human (No. 2020kmykdx6h05). Witten informed consent was obtained from the patient's daughter.

\section{Correlation analysis between OCRT and adverse reactions}

\subsection{Correlation analysis between patient's medical history and tremor}

Tremor is the rhythmic, involuntary vibration of muscles in one or more functional areas, which is the result of abnormal synchronization of motor neurons [6, 7]. Among previous diseases contracted by the patient, respiratory alkalosis and cerebral circulation insufficiency might lead to convulsive tremor of limbs [8]. Although the respiratory alkalosis-induced convulsive tremor is attributed primarily to hypocalcemia, the physical examination of the patient revealed serum $\mathrm{Ca}^{2+}>2.2 \mathrm{mmol} / \mathrm{L}$, suggesting absence of hypocalcemia, so the respiratory alkalosis-induced convulsive tremor was excluded. MRI examination: No apparent abnormalities were found on the craniocerebral 
DWI scans; cerebral function examination: No abnormalities were found. No treatment was given for cerebral circulation insufficiency in the later stage, nor the tremor was relieved, so the cerebral circulation insufficiency-induced tremor was excluded.

\subsection{Correlation analysis between patient's medication history and tremor}

Aside from OCRT, there are also many other drugs that can induce tremor, such as penicillin, cocaine and metoclopramide $[9,10]$. On the 16th day of hospitalization, the tremor in the patient ceased after replacing OCRT with morphine sulfate controlled-release tablets. Thus, the possibility of tremor induced by drugs that were administered after the 16th day could be ruled out. Among the drugs still in use on the 16th day, those could cause adverse reaction of tremor included: Budesonide/formoterol inhaler, terbutaline sulfate injection, metoclopramide tablets and moxifloxacin injection. However, since these drugs were still in use after the tremor stopped, the possibility of tremor caused by them could be ruled out. After excluding the effects of the above drugs and replacing OCRT with morphine sulfate controlled-release tablets, the tremor ceased. The rare adverse reactions of tremor are also described in the instructions of OCRT. A reasonable temporal relationship is present between the OCRT and the occurrence of tremor. According to research on oxycodone-induced convulsions in patients, there are no other factors or drugs that could cause tremor. Hence, it is highly probable that the tremor occurred in the patient was an adverse event caused by oxycodone hydrochloride.

\section{Discussion}

\subsection{Exploration of mechanism for OCRT-induced tremor}

\section{Exploration of mechanism for OCRT-induced tremor based on the locus coeruleus function}

The primary mechanism of action of OCRT is to stimulate the $\mu$ opioid receptors on sensory neuron membranes in the central nervous system, and to block the process of pain stimulus transmission from the unmyelinated nerve fibers to the central nervous system, thereby achieving analgesia [11, 12]. As some experiments on a variety of mammals have proved $[5,7,13,14]$, the $\mu$ receptors in the brain are distributed mainly in the areas related to pain sensation and breathing (as well as nausea and vomiting), such as the trigeminal nucleus, thalamus, locus coeruleus and nucleus ambiguous. It is thus speculated that oxycodone may cause tremor by acting on the locus coeruleus. The bases for relevant hypotheses are as follows:

Hypothesis one: Weakened function of $\gamma$-aminobutyric acid ( $\gamma$-GABA) receptor can cause tremor. According to prior animal experiments, harmaline ( $\beta$-carboline alkaloid analogue) increases the transmission of inferior olivary-cerebellar pathway by inhibiting the $\gamma$-GABA receptor, thereby leading to tremor that resembles essential tremor. There is a projective relationship between locus coeruleus and cerebellar Purkinje cells. Locus coeruleus lesions may cause reduced release of inhibitory neurotransmitter $\gamma$-GABA by the cerebellar Purkinje cells, thereby resulting in tremor $[15,16]$. OCRT may cause lowered $\gamma$-GABA secretion by stimulating the $\mu$ receptors on locus coeruleus, which in turn produces tremor.

Hypothesis two: According to recent studies exploring the roles of locus coeruleus and its major secreted neurotransmitter norepinephrine (NE) in the development and progression of Parkinson's disease (PD), the absence of NE itself can induce significant neuroinflammation in various projecting brain areas of locus coeruleus, which can also lead to a top-down sequential decrease in neurons from the neuroendocrine factor (SN) in the brain to the prefrontal cortex of hippocampus, thereby resulting in weakened dopamine neuron protection. As the in-vitro experiments of mechanism have proven, NE inhibits the production of superoxide neuroinflammatory factors by activating NOX2 on the microglial cells, thereby playing a role in protecting dopamine neurons. Thus, OCRT probably acts on $\mu$ receptors on locus coeruleus, which leads to a decreased NE secretion by the locus coeruleus to weaken the dopamine neuron protection, thereby inducing the PD-like tremor [17].

\section{5-HT syndrome-induced tremor}

5-HT syndrome, as a high 5-HT state induced by drugs, produces symptoms like altered mental status of organisms, neurological dysfunctions of plants and neuromuscular dysfunctions, where neurons are discharged rhythmically to result in tremor. 5-HT also induces excitability in the raphe nuclei of brainstem, thereby leading to myoclonus and tremor [18-22]. Opioid analgesics haven been found to cause excessive 5-HT and lead to 5-HT syndromic tremor. In our report, the patient did not develop such symptom after using morphine sulfate controlled-release tablets. The probable reason is that the morphine in opioid analgesics is a non-serotonergic agent, while oxycodone possesses a 5-HT activity, so the use of oxycodone leads to high 5-HT state to induce tremor. 


\section{Differences in adverse reactions between morphine sulfate and oxycodone hydrochloride}

Although morphine also acts on the $\mu$ opioid receptors, the patient in our case experienced tremor after administration with OCRT, which disappeared after changing the medication to morphine sulfate controlled-release tablets. Regarding the possible reason, oxycodone was easier to cross the blood-brain barrier than morphine due to the structural differences in 3-hydroxyl group between the two agents. Research has demonstrated that only when the plasma drug level of morphine sulfate is several times higher than that of oxycodone hydrochloride [12], can the two drugs reach the same intracerebral drug concentrations. This is why the pharmacological effects and adverse reactions vary between the oxycodone hydrochloride and the morphine sulfate. In our case, the patient was given morphine sulfate controlled-release tablets $60 \mathrm{mg} / \mathrm{d}$ and OCRT $20 \mathrm{mg} / \mathrm{d}$. In view of the only 3-folds higher dose of morphine than oxycodone, the intracerebral drug concentration of morphine sulfate was considerably lower than oxycodone hydrochloride. Possibly, this was the main reason for the difference in adverse reactions.

\section{Explanation of individual differences in adverse reactions between morphine sulfate and oxycodone hydrochlo- ride from the gene polymorphism perspective}

Adverse reactions to the use of opioids vary distinctly among individuals. Such difference in adverse reactions by organisms may be associated with the regulation of opioid pharmacodynamics (receptors) at the gene level. As the major target of opioid analgesia, the diversity of $\mu$-receptor encoding gene OPRM1 causes difference in the reactivity of downstream signaling pathways, which is the primary factor affecting the inter-opioid variability in analgesic effects and adverse reactions. The T802C and A118G mutations of the OPRM1 have been shown to reduce the individual sensitivity to morphine. Thus, the adverse effect of $\mu$-receptor gene polymorphisms on morphine is non-negligible.

In clinical practice, the effective dose, analgesic effect and adverse events of oxycodone vary greatly from individual to individual. According to extant research, such individual variability was probably attributed to the effects of genetic polymorphisms on the pharmacokinetics and pharmacodynamics of oxycodone. To date, the most studied OPMR1 locus is A118G. A study has found that the M6G-induced adverse reactions were fewer among G118 allele carriers than the A118 carriers. As a prior study suggested, the incidence of adverse events increased in the (OPRMI).

C354A mutant group (CG+GG genotype), meanwhile, the therapeutic efficacy was reduced. In a study of OPRM1 genotype polymorphism and analgesic dosage of oxycodone hydrochloride by Li Q et al. [23], the patients carrying allele $\mathrm{G}$ needed more oxycodone hydrochloride for controlling pain. As a result, their demand for opioid agents increased, so did the adverse reactions they developed. Thus, presumably, the differences in adverse reactions between morphine sulfate and oxycodone hydrochloride in the patient reported herein were caused by the gene polymorphism differences among opioid receptors.

\section{Prospects}

In the instructions of OCRT, oxycodone-induced tremor is described as a particularly rare adverse event, which has never been reported in the clinical setting. Despite very low incidence, such adverse reaction needs clinical attention, rather than being ignored. The problem is the great individual differences in the effective analgesic dose, plasma concentration and adverse reactions of opioids for clinical treatment. Genetic-level understanding of the pharmacokinetics and pharmacodynamics enables the clinicians to use opioids in a more reasonable, scientific and economical manner. Hence, genotyping of individual patients is necessary prior to receiving opioid analgesics, so that the precise medication guidance can be given to improve therapeutic effect and lower adverse reactions.

Prior to using oxycodone hydrochloride tablets, it is recommended to choose an appropriate analgesic according to the specific conditions and medical history of patients, in order to reduce the analgesic-induced adverse reactions. Once upon occurrence of an adverse reaction, the analgesic should be discontinued immediately and replaced with another appropriate analgesic.

\section{Statement of Ethics}

All procedures carried out in this study were in accordance with the ethical standards of the institutional and national responsible committee on human experimentation and the Helsinki Declaration of 1964 and its later amendments or equivalents. The study protocol was approved by the Ethics Committee of Sixth Affiliated Hospital of Kunming Medical University on human (Approval number: 2020kmykdx6h05). Witten informed consent for publication was obtained from the patient's daughter. 


\section{Funding Sources}

This work was supported by the National Natural Science Foundation of China (81360204 to ZT).

\section{Authors' Contributions}

Qirui Tai conceived and designed the experiments; Mili Shi performed the experiments; Zhiwei Tang analyzed the data; Qiuyan Song and Yijie Shi wrote the paper. All authors read and approve the final version of the manuscript.

\section{References}

[1] Pöyhiä, R., A. Vainio, E. Kalso. (1993). A review of oxycodone’s clinical pharmacokinetics and pharmacodynamics. J Pain Symptom Manage, 8: 63-67.

[2] Chen, T., H. Wu, C. Zhang. (2018). Literature Analysis on Adverse Drug Reactions caused by Oxycodone in China. Chinese Journal of Pharmacoepidemiology, 27: 53-57.

[3] Hu, J. N. (2014). Delirium induced by oxycodone hydrochloride for cancer pain control: A report and analysis of two cases. Chinese Journal of Pain Medicine, 20: 367-368.

[4] Xu, X., X. Wu, Z. Xue, X. Wang, L. Xiong, M. Tian, C. Yao. (2013). Efficacy and safety of oxycodone hydrochloride injection for postoperative analgesia in patients undergoing operation under general anesthesia: a prospective, randomized, blind, multicenter, positive-controlled, clinical trial. Chinese Journal of Anesthesiology, 269-274.

[5] Cao, Y., C. Zheng, Y. Yang. (2016). Two novel adverse reactions like convulsions caused by acetaminophen and oxycodone tablets. Chinese Journal of Hospital Pharmacy, 36: 785.

[6] Zhang, S. Y., Zhang, T. (2003). Research status of tremor and its mechanism. Chinese Journal of Rehabilitation Theory and Practice: 21-23.

[7] Klein, M., Z. Rudich, B. Gurevich, M. Lifshitz, S. Brill, M. Lottan, N. Weksler. (2005). Controlled-release oxycodone-induced seizures. Clin Ther, 27: 1815-1818.

[8] Yang, L.Y., He, X. Y. (2011). Clinical observation of 222 elderly patients with cardiogenic cerebral ischemia syndrome from high-altitude areas. Chinese and Foreign Medical Research, 09: 95-96.

[9] Chen, W. B., Pan, X. L. (2008). Diagnostics (Seventh Edition). Beijing: People’s Medical Publishing House, 66.

[10] Zhang, X. H., Chen, B. Y. (2014). Sudden syncope and convulsions are not epilepsy Well-Being, 04: 44-45.

[11] Sun, C. J. (2008). A case report of 5-HT syndrome induced by paroxetine. Journal of Psychiatry, 6: 422.

[12] Deuschl, G., Raethjen, J., Baron, R., Lindemann, M., Wilms, H., Krack, P. (2000). The pathophysiology of parkinsonian tremor: a review. J Neurol, 247: V33-V48.

[13] Hardman, J. G. E. L. (2004). Goodman \& Gilman’s The Pharmacological Basis of Therapeutics (10th edition). Beijing: People's Medical Publishing House, 447.

[14] Xu, J. (2014). Pharmacology and clinical application of oxycodone hydrochloride. J Clin Anesthesiol, 30: 511-513.

[15] Benito-León, J., Louis. E. D. (2006). Essential tremor: emerging views of a common disorder. Nat Clin Pract Neurol, 2: 666-678; quiz 662p following 691.

[16] Wu, Y. W., Xiao, Q. (2009). Progress in the research of pathogenesis and treatment of essential tremor. Chinese Journal of Contemporary Neurology and Neurosurgery, 09: 235-237.

[17] Jiang, L. (2015). Locus coeruleus injury promotes the development and progression of Parkinson's disease. Chinese Society of Toxicology: 233.

[18] Martin, T. G. (1996). Serotonin syndrome. Ann Emerg Med., 28: 520-526.

[19] Zafonte, R. D., Cullen, N., Lexell, J. (2002). Serotonin agents in the treatment of acquired brain injury. J Head Trauma Rehabil., 17: 322-334.

[20] Numenthaler, M., Mattle, H. (2004). Neurology. Stuttgart: Thieme, 274: 305-307.

[21] Richards, D., J. Aronson, D. John Reynolds, J. Coleman. (2006). Oxford Handbook of Practical Drug Therapy. Beijing: People's Medical Publishing House, P. 299.

[22] Chen, M. (2010). One case of 5-HT syndrome induced by tramadol hydrochloride injection and paroxetine. Chinese Journal of Pharmacovigilance, 07: 185.

[23] Li, Q., Liu, Y., Yu, Y. (2016). $\mu$ opioid receptor A118G gene polymorphism predicts analgesic effect of oxycodone hydrochloride. Medical Guide, 33: 481-484. 